\section{Elevated UV-B radiation reduces genome stability in plants}

\author{
Gerhard Ries ${ }^{\star}$, Werner Heller $\dagger$, Holger Puchta ${ }^{\star} \ddagger$, \\ Heinrich Sandermann $\dagger$, Harald K. Seidlitz $\uparrow \&$ Barbara Hohn* \\ * Friedrich Miescher Institut, PO Box 2543, CH 4002 Basel, Switzerland \\ $\dagger$ GSF Forschungszentrum für Umwelt und Gesundheit, D 85764 Neuherberg, \\ Germany
}

Long term depletion of the stratospheric ozone layer contributes to an increase in terrestrial solar ultraviolet $B$ radiation $^{13}$. This has deleterious effects on living organisms, such as DNA damage $e^{4,5}$. When exposed to elevated ultraviolet $B$ radiation (UV B; $280315 \mathrm{~nm}$ ), plants display a wide variety of physiologi cal and morphological responses characterized as acclimation and adaptation $^{6}$. Here we show, using special sun simulators, that elevated solar UV B doses increase the frequency of somatic homologous DNA rearrangements in Arabidopsis and tobacco plants. Increases in recombination are accompanied by a strong induction of photolyase and Rad51 gene expression. These genes are putatively involved in major DNA repair pathways, photo reactivation and recombination repair $^{7,8}$. In mutant Arabidopsis plants that are deficient in photoreactivating ultraviolet induced cyclobutane pyrimidine dimers, recombination under elevated UV B regimes greatly exceeds wild type levels. Our results show that homologous recombination repair pathways might be involved in eliminating UV B induced DNA lesions in plants. Thus, increases in terrestrial solar UV B radiation as forecasted for the early 21 st century may affect genome stability in plants.

The stratospheric ozone layer is the key factor in reducing solar UV B radiation reaching the Earth's surface ${ }^{3}$. The continuous depletion of the ozone layer will, therefore, be accompanied by an increase in terrestrial UV B irradiance, especially at wavelengths

‡ Present address: IPK, Institut für Pflanzengenetik und Kulturpflanzenforschung, D-06466 Gatersleben, Germany. below $300 \mathrm{~nm}^{13,5}$. Whereas the impact of UV B radiation on plant growth, development, physiology and morphology has been studied intensively ${ }^{9}$, little is known about its influence on plant genome stability. Homologous recombination is particularly important to plants as their genomes contain large amounts of repeated DNA sequences and highly homologous gene families ${ }^{10}$. In addition, rearrangements between homologous DNA sequences in somatic cells are strongly stimulated by DNA damaging agents ${ }^{10}$.

To obtain a realistic estimate of the long term impact of increased solar UV B irradiance on plant genomes, we exposed Arabidopsis and tobacco plants over several generations to different levels of elevated UV B radiation. Genome stability was monitored by assaying homologous recombination in treated plants and their offspring. To simulate the natural photobiological environment we provided a natural spectral balance of photosynthetic active radia tion (PAR; $400700 \mathrm{~nm}$ ) of $800 \mu \mathrm{mol} \mathrm{m}^{-2} \mathrm{~s}^{-1}$ and ultraviolet A radiation (UV A; $315400 \mathrm{~nm}$ ) of $19 \mathrm{Wm}^{-2}$, conditions under which photobiological processes are activated to normal physio logical levels ${ }^{11}$. The spectrum was supplemented with four different UV B regimes ${ }^{12}$ which are given as biologically effective (BE) weighted daily UV B dose normalized at $300 \mathrm{~nm}^{13}$ (details on irradiation spectra and an alternative weighting are available as Supplementary Information). The 'ambient' UV B regimes, UV B1 $\left(2.3 \mathrm{~kJ} \mathrm{~m}^{-2} \mathrm{~d}_{\mathrm{BE}}^{-1}\right)$ and UV B2 $\left(6.6 \mathrm{~kJ} \mathrm{~m}^{-2} \mathrm{~d}_{\mathrm{BE}}^{-1}\right)$, lie in the range of UV B currently encountered during the growing period in northern mid latitudes $\left(40^{\circ} \mathrm{N} 60^{\circ} \mathrm{N}\right)$ at altitudes up to about $1,000 \mathrm{~m}$ above sea level. Two 'high' regimes, UV B3 $\left(18.6 \mathrm{~kJ} \mathrm{~m}^{-2} \mathrm{~d}_{\mathrm{BE}}^{-1}\right)$ and UV B4 $\left(27.1 \mathrm{~kJ} \mathrm{~m}^{-2} \mathrm{~d}_{\mathrm{BE}}^{-1}\right)$ were used to test the plant's response to strongly elevated UV B radiation.

The influence of increased UV B radiation on somatic recombi nation frequency was analysed using one transgenic Nicotiana tabacum line and four independent Arabidopsis thaliana lines, carrying one or more copies of a recombination reporter transgene at a single but different locus each. All recombination substrates contain two overlapping parts of the $\beta$ glucuronidase gene which are orientated as tandem or inverted repeats and are interrupted by a hygromycin resistance gene. Homologous recombination between these overlapping sequences leads to a complete, functional $\beta$ glucuronidase gene. The length of overlap between the two homo

Table 1 Somatic recombination frequency per genome $\left(\times 10^{6}\right)$ in transgenic Arabidopsis and tobacco plants

\begin{tabular}{|c|c|c|c|c|c|}
\hline \multirow[b]{2}{*}{$\begin{array}{l}\text { Daily UV B dose } \\
\left(\mathrm{kJ} \mathrm{m}{ }^{2} \mathrm{~d}^{1}\right)_{\mathrm{BE}}\end{array}$} & \multicolumn{5}{|c|}{ Line } \\
\hline & N9 & A11 & A211 & A231 & A651 \\
\hline 2.3 (UV B1) & $11.3 \pm 2.1$ & $35.3 \pm 4.9$ & $0.6 \pm 0.1$ & $0.1 \pm 0.0$ & $1.1 \pm 0.1$ \\
\hline 6.6 (UV B2) & $52.3 \pm 8.2$ & $58.6 \pm 6.2$ & $2.8 \pm 0.5$ & $0.2 \pm 0.1$ & $2.2 \pm 0.3$ \\
\hline 18.6 (UV B3) & n.d. & $70.9 \pm 9.2$ & $5.1 \pm 0.7$ & $0.5 \pm 0.1$ & $4.4 \pm 0.5$ \\
\hline 27.1 (UV B4) & $83.5 \pm 17.1$ & $86.8 \pm 13.3$ & $8.4 \pm 1.0$ & $0.8 \pm 0.2$ & $6.9 \pm 0.6$ \\
\hline \multirow{2}{*}{$\begin{array}{l}\text { Daily UV B dose } \\
\left(\mathrm{kJ} \mathrm{m}{ }^{2} \mathrm{~d}^{1}\right)_{\mathrm{BE}}\end{array}$} & \multicolumn{2}{|c|}{ Line } & & & \\
\hline & A87 & A94 & & & \\
\hline 2.3 & $0.5 \pm 0.1$ & $0.9 \pm 0.1$ & & & \\
\hline 7.7 & $1.2 \pm 0.2$ & $6.5 \pm 1.1$ & & & \\
\hline \multirow[t]{3}{*}{13.4} & $2.2 \pm 0.4$ & $25.1 \pm 2.9$ & & & \\
\hline & \multicolumn{3}{|c|}{$\begin{array}{l}\text { Generation } \\
\text { (Line A651) }\end{array}$} & & \\
\hline & $\mathrm{F}_{0}$ & $F_{1}$ & $\mathrm{~F}_{2}$ & & \\
\hline 2.3 & $1.3 \pm 0.2$ & $1.3 \pm 0.2$ & $1.4 \pm 0.3$ & & \\
\hline 6.6 & $2.4 \pm 0.3$ & $5.7 \pm 0.8$ & $7.7 \pm 1.1$ & & \\
\hline 18.6 & $4.9 \pm 0.7$ & $12.3 \pm 1.8$ & $15.7 \pm 2.2$ & & \\
\hline
\end{tabular}

Each value represents the mean of $80-120$ plants \pm s.e. The structure of the recombination Each value represents the mean of $80-120$ plants \pm S.e. The structure of the recombination substrates for the different lines has been described ${ }^{1}$. In brief, the tobacco line N9 carries four copies of the recombination substrate in indirect orientation, with 566-base pair (bp) homologous overlapping sequence; the Arabidopsis line A11 contains one copy of the recombination substrate in direct orientation, with 1,213-bp overlap; the Arabidopsis lines A211 (one copy) and A231 (two copies) carry the recombination substrates in direct orientation with 566-bp overlap (P. Swoboda, personal communication), and line A651 one copy in indirect orientation, with 566-bp overlap. n.d. not determined. 


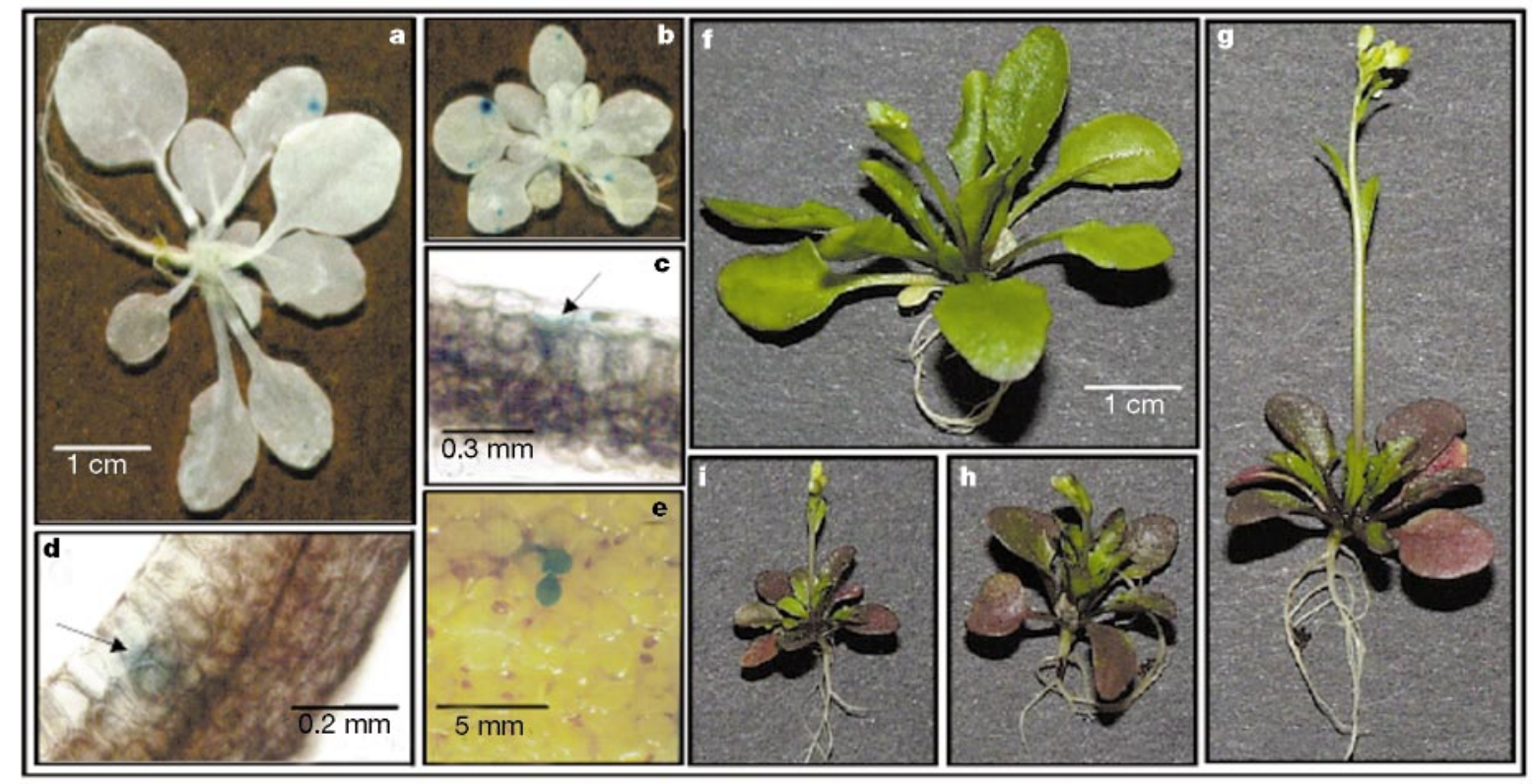

Figure 1 Transgenic Arabidopsis thaliana plants (line A11) grown under different daily UV B doses. a, b, Visualization by histochemical staining of somatic recombination events in 23 day old plants exposed to UV B1 (a) and UV B4 (b). The scale is the same for both. c, d, Sections through stained leaves from plants exposed to UV B4. Arrows indicate recombination events. e, Germinal recombination event in an $F_{1}$ population of 7 day old

logous sections and their orientation to each other varies between recombination substrates ${ }^{14,15}$ (Table 1). After histochemical staining of plants, recombination events can be visualized and their number and location determined (Fig. la e).

In all the lines tested, the somatic recombination frequency under the current ambient UV B1 level ranged from $(1.0 \pm 0.2) \times 10^{7}$ to $(3.5 \pm 0.5) \times 10^{5} \quad$ (mean \pm s.e.) recombination events per genome (Table 1). The large variations between the lines may result from differences in the structure of the recombination substrates, transgene copy number and plant species. Differences in the genomic position of the randomly integrated recombination substrates may also be an important factor influencing both transcriptional activity and recombination frequency ${ }^{15,16}$.

Exposure to different levels of elevated UV B radiation induced morphological changes (Fig. If i) and markedly affected the somatic recombination frequency in all the plant lines tested (Table 1). Under the elevated but still ecologically relevant UV B2 regime the recombination frequency in tobacco and all Arabidopsis lines increased significantly $(P<0.05, t$ test $)$. The recombination frequencies in line N9 rose 4.6 fold, in line A11 1.7 fold, in line A211 4.6 fold and in lines A231 and A651 twofold. With the higher UV B doses, UV B3 and UV B4, recombination was affected even more (up to 8.5 fold increases between UV B1 and UV B3 and up to 14 fold increases between UV B1 and UV B4). It seems that, in general, the induction of recombination under elevated UV B radiation is independent of the plant species and line analysed and is probably a genome wide phenomenon with varying specifi city for different loci. The formation and position of UV induced damage in DNA, as well as the accessibility and activity of DNA repair processes, are influenced largely by chromatin structure, at

Table 2 Germinal recombination frequency in Arabidopsis plants of line A11

\begin{tabular}{|c|c|}
\hline $\begin{array}{l}\text { Daily UV B dose } \\
\left(\mathrm{kJ} \mathrm{m}^{2} \mathrm{~d}^{1}\right)_{\mathrm{BE}}\end{array}$ & $\begin{array}{l}\text { Germinal recombination } \\
\text { (events per } 250,000 \text { seedlings) }\end{array}$ \\
\hline 2.3 (experiment 1 ) & 1 \\
\hline 2.3 (experiment 2) & 2 \\
\hline 6.6 & 4 \\
\hline 18.6 & 10 \\
\hline
\end{tabular}

seedlings grown under UV B3 regime. The $\mathrm{F}_{0}$ generation was grown under the identical UV B3 regime. $\mathbf{f}$ i, 23 day old Arabidopsis, germinated and grown under $41 \mathrm{~mol} \mathrm{~m}^{-2} \mathrm{~d}^{-1}$ photosynthetic active radiation with different daily UV B doses. $\mathbf{f}$, UV B1 $=$ $2.3 \mathrm{~kJ} \mathrm{~m}^{-2} \mathrm{~d}^{-1}{ }_{\mathrm{BE}} ; \mathbf{g}$, UV B2 $=6.6 \mathrm{~kJ} \mathrm{~m}^{-2} \mathrm{~d}^{-1}{ }_{\mathrm{BE}} ; \mathbf{h}$, UV B3 $=18.6 \mathrm{~kJ} \mathrm{~m}^{-2} \mathrm{~d}^{-1}$ and $\mathbf{i}$, UV B4 $=27.1 \mathrm{~kJ} \mathrm{~m}^{-2} \mathrm{~d}^{-1}{ }_{\mathrm{BE}}$. Scale is the same for $\mathbf{f}-\mathbf{i}$.

least in yeast and mammalian cells ${ }^{17,18}$. Different loci in the plant genome may also respond to UV B stress with different increases in recombination activity.

Increased levels of recombination under elevated UV B radiation may be an immediate consequence of increased damage and repair of affected DNA. To test whether repair of UV B induced DNA damage influences recombination, we crossed the transgenic Arabidopsis line A651 with the photolyase deficient line uvr2 1 (ref. 19), which lacks photoreactivation repair of cyclobutane pyrimidine dimers, the major UV B induced DNA damage. In the resulting progeny line A94, homozygous for $u v r 21$, the presence of UV $B$ radiation generally stimulated homologous recombination to a greater extent than in the control progeny line A87, homozygous for UVR2 1 (Table 1). In UV B1, the recombination frequency in line A94 was already about 1.8 fold higher than in line A87. Photolyase deficient plants were much more sensitive to UV B radiation than wild type plants, therefore, we applied slightly reduced regimes. A daily dose of $7.7 \mathrm{~kJ} \mathrm{~m}^{-2} \mathrm{~d}^{-1}$ BE UV B induced recombination in line A94 5.4 times more strongly than in line A87 and a dose of $13.4 \mathrm{~kJ} \mathrm{~m}^{-2} \mathrm{~d}^{-1}{ }_{\mathrm{BE}} \mathrm{UV} \mathrm{B}$ increased the recombination frequency up to 11.4 times more efficiently than in line A87. Higher UV B doses were lethal for photolyase deficient plants. The absence of cyclobutane pyrimidine dimer specific photoreactivation repair increased the steady state concentration of cyclobutane pyrimidine dimers in mutants exposed to elevated UV B levels, compared with wild type plants (Fig. 2a). In contrast, the concentration of (6 4) photoproducts was unaffected under elevated UV B regimes in photolyase mutant plants, as in wild type plants (data not shown). These data indicate that there may be a strong relationship between cyclobutane pyrimidine dimers in the plants' genomes and homologous recombination, and furthermore that recombina tion might be involved in cyclobutane pyrimidine dimer repair. Elimination of UV B induced DNA lesions, by DNA repair pathways such as nucleotide excision, may create recombinogenic intermediates. Alternatively, or in addition, UV B promoted signalling pathways may activate recombination processes by stimulating, for example, transcription of DNA repair genes ${ }^{20}$ (see below). 
Plants may compensate for deleterious effects of elevated UV B radiation by mounting various acclimation responses, including accumulation of secondary metabolites and stimulation of DNA repair ${ }^{6}$. The accumulation of UV B screening compounds, mainly flavonols, is involved in protecting against UV B radiation ${ }^{21,22}$. We therefore determined the concentration of quercetin and kaempferol derivatives (Fig. 2b) as well as sinapate esters and anthocyanin compounds in UV B treated plant populations. In plants exposed to the UV B2 level, the production of flavonols was significantly induced $(P<0.01, t$ test $)$ compared with UV B1 treated plants. Under UV B3 or UV B4 conditions, only a weak additional flavonol accumulation could be observed (Fig. 2b) Unlike flavonols, sinapate esters were not induced by elevated UV B radiation (data not shown). The content of the visible anthocyanins was increased under higher UV B levels (Fig. If i), although to much lower levels than those of flavonols (data not shown), indicating a minor role for the anthocyanins in absorbing UV B. Under the anticipated elevated UV B levels the protection against UV B radiation afforded by UV B screening pigments may therefore be incomplete.

This may, at least in part, be counterbalanced by enhanced repair activity. We compared the repair activities in plants exposed to the different UV B regimes by examining the steady state levels of AtPhotolyase II (PHR1) and AtRad51 messenger RNA, the latter as a putative marker for recombination repair. Using polymerase chain reaction with reverse transcriptase (RT PCR), we found that the steady state mRNAs of both genes were increased under high UV B doses (Fig. 2c). This suggests that plants grown under elevated UV B regimes have an increased ability to repair DNA damage. This ability may reduce at least DNA damage resulting in cyclobutane pyrimidine dimers and (6 4) photoproducts to the level of that found in plants exposed to ambient UV B conditions (Fig. 2a). In animals and yeast, Rad51 is crucial for recombination ${ }^{8,23}$ and in plant cells it has been reported to be strongly induced after gamma irradiation ${ }^{24}$. If AtRad51 is involved in recombination

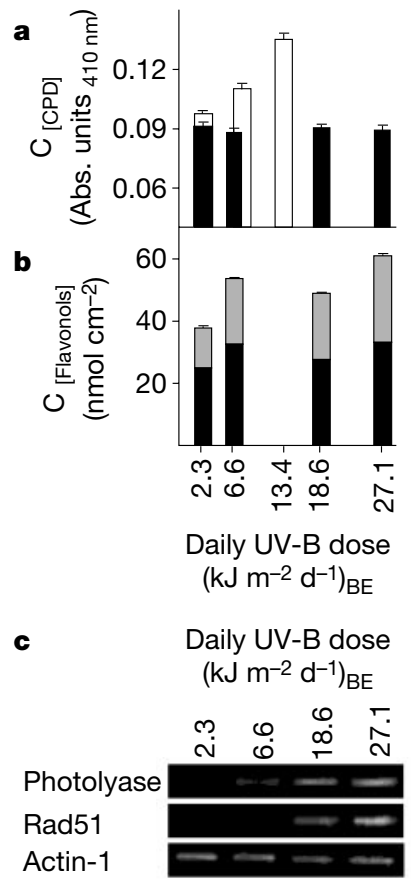

Figure 2 UV B acclimation responses in Arabidopsis thaliana line A651 grown under different daily UV B doses. a, Cyclobutane pyrimidine dimer (CPD) concentrations in wild type (black bars) and photolyase deficient plants (white bars). Vertical bars represent s.e. b, Flavonol concentrations, measured by quercetin (grey bars) and kaempferol (black bars) derivatives. Vertical bars represent s.d. c. Steady state mRNA levels for photolyase ॥ (PHR1), AtRad51 protein and AtActin 1 determined by RT PCR. repair in plants, enhanced expression of AtRad51 under elevated UV B levels could result in higher recombination repair activity, which in turn may contribute to increased frequencies of homologous recombination.

Germinal recombination events can permanently alter the genetic composition of subsequent generations and hence contribute to genomic changes in plant populations ${ }^{25}$. Although reproductive organs are generally considered to be well protected from UV B during both developmental and mature phases, long term exposure to high UV B levels may also affect the reproductive tissue (L2 cell layer) of plants and cause DNA damage. Mature pollen grains are potentially very susceptible to UV B induced DNA damage during the short period between anther dehiscence and pollen tube penetration into stigma tissues ${ }^{26}$. Indeed, UV B irradiation of maize pollen can activate cryptic transposable elements ${ }^{27}$. Recom bination processes may also be induced under such conditions, affecting the genome stability of future plant generations. We analysed the influence of UV B radiation on the frequency of germinal recombination in Arabidopsis plants. Germinal recombi nants are relatively rare in plants and can be the products of meiotic recombination or representatives of somatic homologous interactions ${ }^{16}$. To detect such rare germinal recombinants, we screened large numbers of seedlings of the subsequent generation of plants grown under elevated UV B regimes. Compared to UV B1 conditions, UV B2 regimes increased the number of germinal recombinations in seven day old Arabidopsis seedlings about four times and UV B3 regimes up to ten times (Fig. 1e, Table 2). The germination rate and the seed set did not differ between the analysed plant populations (data not shown). Although the numbers of germinal recombination events were small, this pre liminary experiment may indicate that elevated UV B levels can lead not only to an increase in genomic instability of somatic tissue but also to permanent changes in plant populations. Furthermore, the progeny of plants exposed to elevated UV B exhibited a higher UV $B$ induced somatic recombination rate than the parental popula tion. Whereas ambient UV B1 levels did not stimulate somatic recombination in subsequent plant generations, exposure of popu lations to UV B2 and UV B3 resulted in a marked increase in the recombination frequency in progeny plants (Table 1). When exposed to similar UV B2 levels as the parental plants, the $F_{1}$ progeny showed a 2.4 fold and the $F_{2}$ progeny a 3.2 fold higher recombination frequency than $\mathrm{F}_{0}$ plants. Under UV B3 regimes the recombination frequencies in the $F_{1}$ and the $F_{2}$ generation increased by nearly the same factors of 2.5 and 3.2, respectively. Thus, elevated UV B exposure over several generations may lead to progressive increases in somatic recombination rates as well as to higher numbers of permanently altered plants.

Under natural conditions the terrestrial UV B irradiance varies strongly depending on the thickness of the UV screening ozone layer, the solar elevation and meteorological conditions. Therefore, extrapolations from data obtained under experimental, static UV B conditions to natural, dynamic UV B field conditions are difficult. Our data, however, allow extrapolations on the entire ecologically relevant UV $B$ range because all recombination data obtained under the 'high' UV B regimes (UV B3, UV B4) are in line with the results obtained under the currently ecological UV B range (UV B1, UV B2). We believe, therefore, that the increase in solar UV B radiation, due to a long term depletion of the stratospheric ozone layer, may influence the genomic stability of plant populations.

\section{Methods}

\section{UV spectra}

A combination of four lamp types (metal halide lamps, quartz halogen lamps, blue fluorescent tubes and UV-B fluorescent tubes) was used to obtain a natural balance of simulated global radiation throughout the ultraviolet to infrared spectrum. The short-wave cut-off was shaped by selected borosilicate, soda-lime and acrylic glass sheets $^{12}$. The mean PAR was $800 \mu \mathrm{molm}^{2} \mathrm{~s}^{1}$, which corresponds to a daily dose of 
$\sim 41 \mathrm{~mol} \mathrm{~m}^{2} \mathrm{~d}^{1}$ PAR. The calculations for the UV-B doses were performed with the general plant action spectrum normalized at $300 \mathrm{~nm}^{13}$. For comparison, we also provide the UV levels weighted with the action spectrum for dimer formation also normalised at $300 \mathrm{~nm}$ (dimer) ${ }^{28}$ (for details see Supplementary Information). ${ }^{11}$ The ratio (UV-B:PAR) is based on the non-weighted irradiance in the PAR and UV-B ranges. The spectroradiometric measurements were performed with a double monochromator system ${ }^{12}$. Variations of the integrated values were less than $15 \%$. We kept the UV-A irradiance constant at $\sim 19 \mathrm{Wm}^{2}$ (non-weighted value) which corresponds to the moderate PAR value. Under the UV-B1 regime, the simulated UV-B:UV-A:PAR ratio of 1:50:480 (rounded figures) closely approximates an average present-day outdoor value around noon of 1:47:400 in northern mid-latitudes under an ozone column of $\sim 320$ Dobson units (typical ratio measured at Neuherberg $\left(48^{\circ} 41^{\prime} \mathrm{N}, 11^{\circ} 41^{\prime} \mathrm{E}\right)$, South Germany, $495 \mathrm{~m}$ above sea level).

\section{Growth conditions}

Plants were germinated in soil and grown under $800 \mu \mathrm{mol} \mathrm{m}{ }^{2} \mathrm{~s}{ }^{1} \mathrm{PAR}$ and different UV-B regimes. All climate parameters $\left(14 \mathrm{~h}\right.$ light at $20^{\circ} \mathrm{C}, 10 \mathrm{~h}$ dark at $16^{\circ} \mathrm{C}, 70 \%$ humidity) were identical for all ultraviolet treatments.

\section{Somatic recombination frequency}

23-day-old transgenic plants were stained histochemically and the recombination frequencies per genome were calculated ${ }^{14}$. In photolyase-deficient plants the recombination frequency was analysed 18 days after germination.

\section{Germinal recombination frequency}

Germinal recombination was determined in Arabidopsis seedlings of line A11. The $\mathrm{F}_{0}$ generation was germinated and grown under UV-B1, UV-B2 or UV-B3 until seeds could be harvested. For each UV-B condition around 250,000 seeds were sown on wet filter paper placed on soil. After seven days exposure to similar UV-B regimes as progenitor plants, the seedlings were histochemically stained and the number of fully stained seedlings determined.

\section{Crossings}

Arabidopsis photolyase mutant $(u v r 2-1)^{19}$ was crossed with line A651 and independent lines were generated. $\mathrm{F}_{3}$-generation lines A94 (homozygous for $u v r 2-1$ ) and A87 (homozygous for UVR2-1) are both homozygous for line A651 recombination substrate.

\section{Dimer concentration}

CPDs and (6-4) photoproducts were quantified ${ }^{29}$ in 23-day-old wild-type plants and 18 day-old photolyase-deficient plants. DNA was extracted from $100 \mathrm{mg}$ plant tissue. CPD and (6-4) photoproduct-specific monoclonal antibodies were purchased from Kamiya Biomedical Company. Samples were measured in quadruplicate and compared by analysis of variance within each experiment, using the computer program STATISTICA.

\section{Flavonol concentration}

Quercetin and kaempferol derivatives were analysed ${ }^{30}$ in 23 -day-old plants as used in Fig. 2a. Samples were analysed in duplicate or triplicate within each experiment.

\section{Steady state mRNA levels}

We used $\sim 100 \mathrm{mg}$ of 23-day-old plants, which were germinated and grown under the indicated conditions, to prepare total RNA using standard protocols. Reverse transcription was performed according to the protocol of the manufacturer (Pharmacia) for RT beads using $1 \mu \mathrm{g}$ of total RNA. The complementary DNA was diluted 1:100, and $1 \mu \mathrm{l}$ was used for PCR with specific primers. The primer sequences were for AtPhotolyase II (PHR1) (AF053365) 5'-GCCGTCGTTTTCAATCTGTT-3' and 3'-AATCGATTTGGTGGTGAAGC-5'; for AtRad51 (U43528) 5' -CGTTGAGGAAAGGAAGAGCA-3' and 3'-GTGGCCAAAAACATC AATCC-5'; and for AtActin-1 (M20016) 5' -AAAGGATGCTTATGTTGGCG-3' and 3'AGCCACATACATAGCAGGGG-5'. Products with predicted sizes of 103 base pairs (bp) for AtPhotolyase, $132 \mathrm{bp}$ for AtRad51 and $259 \mathrm{bp}$ for AtActin-1 were obtained. The actin transcript was used as constitutive control. The cDNAs for all tested genes were amplified using hot start PCR under the following conditions: $94^{\circ} \mathrm{C}$ for $5 \mathrm{~min}$; followed by 40 cycles for AtRad51, 35 cycles for photolyase and 30 cycles for AtActin- 1 of $94^{\circ} \mathrm{C}$ for $30 \mathrm{~s}, 60^{\circ} \mathrm{C}$ for $30 \mathrm{~s}$, and $72{ }^{\circ} \mathrm{C}$ for $90 \mathrm{~s}$; ending with $10 \mathrm{~min}$ at $72^{\circ} \mathrm{C}$. Equal loading of each amplified gene sequence was determined by the control AtActin-1 PCR product.

1. Kerr, J. \& McElroy, C. Evidence for large upward trends of ultraviolet-B radiation linked to ozone depletion. Science 262, 1032-1034 (1993).

2. Madronich, S., McKenzie, R. L., Björn, L. O. \& Caldwell, M. M. Changes in biologically active ultraviolet radiation reaching the earth's surface. J. Photochem. Photobiol. B 46, 5-19 (1998).

3. McKenzie, R., Connor, B. \& Bodeker, G. Increased summertime UV radiation in New Zealand in response to ozone loss. Science 285, 1709-1711 (1999).

4. Rozema, J., van de Staaij, J., Björn, L. O. \& Caldwell, M. M. UV-B as an environmental factor in plant life: stress and regulation. Trends Ecol. Evol. 12, 22-28 (1997).

5. Rousseaux, M. C. et al. Ozone depletion and UVB radiation: Impact on plant DNA damage in southern South America. Proc. Natl Acad. Sci. USA 96, 15310-15315 (1999).

6. Jansen, M. A. K., Gaba, V. \& Greenberg, B. M. Higher plants and UV-B radiation: balancing damage, repair and acclimation. Trends Plant Sci. 3, 131-135 (1998).

7. Britt, A. B. Molecular genetics of DNA repair in higher plants. Trends Plant Sci. 4, 20-24 (1999).

8. Vispe, S., Cazaux, C., Lesca, C. \& Defais, M. Overexpression of Rad51 protein stimulates homologous recombination and increases resistance of mammalian cells to ionizing radiation. Nucleic Acids Res. 26, 2859-2864 (1998).

9. Tevini, M. in UV-B Radiation and Ozone Depletion: Effects on Humans, Animals and Plants, Microorganisms and Materials (ed. Tevini, M.) 125-153 (CRC Press, Boca Raton: Lewis Publishers, USA, 1993).

10. Puchta, H. \& Hohn, B. From centiMorgans to base pairs: homologous recombination in plants. Trends Plant Sci. 1, 340-348 (1996).

11. Caldwell, M. M., Flint, S. \& Searles, P. S. Spectral balance and UV-B sensitivity of soybean: a field experiment. Plant Cell Environ. 17, 267-276 (1994).

12. Döhring, T., Köfferlein, M., Thiel, S. \& Seidlitz, H. K. Spectral shaping of artificial UV-B irradiation for vegetation stress research. J. Plant Physiol. 148, 115-119 (1996).

13. Caldwell, M. M. in Photophysiology Vol. 6 (ed. Giese, A. C.) 131-177 (Academic, New York, 1971).

14. Swoboda, P., Gal, S., Hohn, B. \& Puchta, H. Intrachromosomal homologous recombination in whole plants. EMBO J. 13, 484-489 (1994).

15. Puchta, H., Swoboda, P., Gal, S., Blot, M. \& Hohn, B. Somatic intrachromosomal homologous recombination events in populations of plant siblings. Plant Mol. Biol. 28, 281-292 (1995).

16. Tovar, J. \& Lichtenstein, C. Somatic and meiotic chromosomal recombination between inverted duplications in transgenic tobacco plants. Plant Cell 4, 319-332 (1992).

17. Gale, J. M. \& Smerdon, M. J. UV induced (6-4) photoproducts are distributed differently than cyclobutane dimers in nucleosomes. Photochem. Photobiol. 51, 411-417 (1990).

18. Suter, B., Livingstone-Zatchej, M. \& Thomas, F. Chromatin structure modulates DNA repair by photolyase in vivo. EMBO J. 16, 2150-2160 (1997).

19. Landry, L. G. et al. An Arabidopsis photolyase mutant is hypersensitive to ultraviolet-B radiation. Proc. Natl Acad. Sci. USA 94, 328-332 (1997).

20. Mount, D. W. Reprogramming transcription. Nature 383, 763-764 (1996).

21. Stapleton, A. E. \& Walbot, V. Flavonoids can protect maize DNA from the induction of ultraviolet radiation damage. Plant Physiol. 105, 881-889 (1994).

22. Landry, L. G., Chapple, C. C. \& Last, R. L. Arabidopsis mutants lacking phenolic sunscreens exhibit enhanced ultraviolet-B injury and oxidative damage. Plant Physiol. 109, 1159-1166 (1995).

23. Shinohara, A., Ogawa, H. \& Ogawa, T. Rad51 protein involved in repair and recombination in $S$. cerevisiae is a RecA-like protein. Cell 69, 457-470 (1992).

24. Doutriaux, M. P., Couteau, F., Bergounioux, C. \& White, C. Isolation and characterisation of the RAD51 and DMC1 homologs from Arabidopsis thaliana. Mol. Gen. Genet. 257, 283-291 (1998).

25. Walbot, V. On the life strategies of plants and animals. Trends Genet. 1, 165-169 (1985).

26. Jackson, J. F. DNA repair in pollen. A review. Mutat. Res. 181, 17-29 (1987).

27. Walbot, V. UV-B damage amplified by transposons in maize. Nature 397, 398-399 (1999).

28. Quaite, F. E., Sutherland, B. M. \& Sutherland, J. C. Action spectrum for DNA damage in alfalfa lowers predicted impact of ozone depletion. Nature 358, 576-578 (1992).

29. Stapleton, A. E., Mori, T. \& Walbot, V. A simple and sensitive antibody-based method to measure UVinduced DNA damage in Zea mays. Plant Mol. Biol. Reporter 11, 230-236 (1993).

30. Turunen, M. et al. The effects of UV-B exclusion on the soluble phenolics of young Scots pine seedlings in the Subarctic. Environ. Pollut. 106, 219-228 (1999).

\section{Acknowledgements}

We thank C. Langebartels, T. Boller, J. Lucht, G. Buchholz, H. Frohnmeyer, P. Crouzet, P. Pelczar, C. Korner and A. Kuttenberger for discussions and comments. We also thank S. Stich, V. Gloeckler and C. Ramos for assistance. The photolyase mutant line (uvr2-1) was obtained from the Arabidopsis Biological Resource Center, Ohio, USA. This work was supported by a fellowship from the Foundation of the Chemical Industry of Basel to G.R. and Novartis Research Foundation.

Correspondence and requests for materials should be addressed to G.R.

(e-mail: ries@fmi.ch). 


\section{Repository KITopen}

Dies ist ein Postprint/begutachtetes Manuskript.

Empfohlene Zitierung:

Puchta, $\mathrm{H}$.

Gene replacement by homologous recombination in plants.

2002. Plant molecular biology, 48

doi:10.5445/IR/28102002

Zitierung der Originalveröffentlichung:

Puchta, $\mathrm{H}$.

Gene replacement by homologous recombination in plants.

2002. Plant molecular biology, 48 (1-2), 73-182.

doi:10.1023/A:1013761821763 